\title{
Comparison between Different Risk Scores in Predicting Hospital Mortality for Patients with Decompensated Liver Cirrhosis: A Single Center Study
}

\author{
MAHMOUD H. ALLAM, M.D.*; MOHAMED A.S. KOHLA, M.D.* and WESAM S. MORAD, M.D.** \\ The Departments of Hepatology \& Gastroenterology* and Epidemiology \& Preventive Medicine**, \\ National Liver Institute, Menoufia University, Shebin El-Kom, Egypt
}

\begin{abstract}
Background: Predicting prognosis of patients with decompensated liver cirrhosis remains an important issue.

Aim of Study: This study aimed to evaluate the predictive value of different patients' characteristics and risk scores as regard patients' outcome (death or survival).

Patients and Methods: 672 patients with different complications of cirrhosis (gastrointestinal bleeding, hepatic encephalopathy, SBP, HRS and ascites) who were admitted to the National Liver Institute Hospital between 2015 and 2018 were included in this study. On admission to the hospital, assessment of patients by routine laboratory work-up (liver and kidney function tests and complete blood count), ultrasound of the abdomen, upper endoscopy for those with bleeding and ascitic fluid sampling for those with ascites. MELD, MELD$\mathrm{Na}, \mathrm{CTP}, \mathrm{ALBI}$ and PALBI grades were calculated for all patients. At the end of hospitalization, patients outcomes were stratified as survived or died. Patients clinical and laboratory parameters were correlated with hospital mortality. Receiver Operating Curves (ROC) for risk scores were plotted to discriminate the predictive power of risk scores versus mortality.
\end{abstract}

Results: The overall hospital mortality was $27.1 \%$. Univariate analysis revealed age, total and direct bilirubin, albumin, transaminases, alkaline phosphatase, INR, serum creatinine, BUN, serum sodium and white cells count as significant, while multivariate analysis concluded age, total bilirubin, serum creatinine, Blood Urea Nitrogen (BUN), serum sodium $(\mathrm{Na})$, INR and white blood cells count significance with hospital mortality, $(p<0.05)$. The area under receiver operating curve for MELD-Na was (0.85), MELD score (0.84), CTP score (0.82), AlBI grade (0.75) and PALBI grade (0.75), $p<0.001$.

Conclusion: MELD-Na and MELD scores are good predictors for prognosis of patients with decompensated liver cirrhosis. But, ALBI and PALBI grade are promising scores which needs more validation.

Correspondence to: Dr. Wesam S. Morad, E-Mail: wesammorad@gmail.com
Key Words: Decompensated cirrhosis - Predictor scores Survival-HCV.

\section{Introduction}

LIVER cirrhosis is one of the leading causes of morbidity and mortality worldwide and the second cause of digestive disease mortality [1]. It accounts for about 40,000 deaths per year in the United States and about 170,000 deaths per year in Europe where 20 million people suffer of chronic liver diseases [2-4]. The annual rate of liver decompensation is estimated to be about $11 \%$ per year which reduces expected patients' survival to $81.8 \%$ and $50.8 \%$ at $1 \& 5$ years respectively $[\mathbf{1 , 5 , 6 ]}$.

Over time, there was many attempts to develop simple, accurate and objective scoring systems which can predict prognosis of patients with cirrhosis on basis of their clinical and laboratory data $\left.{ }^{7}\right]$.

The Child-Pugh (CTP) score is one of the oldest scores that's widely used to determine prognosis of patients with liver cirrhosis. But, its accuracy is influenced by subjectivity of hepatic encephalopathy and ascites as variables used for its calculation $[8,9]$.

\footnotetext{
Abbreviations:

HCV : Hepatitis C.

MELD : Model for End Stage Liver Disease.

ROC : Receiver Operating Curve.

UGIB : Upper Gastrointestinal Bleeding.

HE : Hepatic Encephalopathy.

HRS : Hepatorenal Syndrome.

SBP : Spontaneous Bacterial Peritonitis.
} 
The Model for End-Stage Liver Disease (MELD) had been developed to estimate survival of patients undergoing trans-jugular intrahepatic Porto-systemic shunts and then was generalized to patients with different etiologies and severity of liver diseases [10]. It was assumed that MELD score is better than CTP score on prediction of waiting-list mortality before liver transplantation

However, mortality still occurs in about $20 \%$ of patients with low MELD score as it was mainly designed for patients with end-stage liver cirrhosis $[12]$.

Other limitations of MELD score was that important complications which may contribute to poor prognosis like ascites and hyponatremia are not included in MELD score. So, many trials were done to modify MELD score in order to improve its predictive accuracy $[\mathbf{1 2 , 1 3 ]}$.

Recently, MELD-Sodium score (MELD-Na) score has been developed to provide better prediction of mortality among patients on the waiting list for liver transplantation (TX) [14,15]

More recently, a new score has been developed to predict survival in patients with Hepatocellular Carcinoma (HCC) based on two variables: Serum albumin and total bilirubin. This score is called the Albumin-Bilirubin (ALBI) grade, a simple score calculated from 2 objective variables which are easily measured and routinely requested for such patients [16]

ALBI grade had significant predictive value for the long-term prognosis of patients with $\mathrm{HBV}$ related cirrhosis (compensated and decompensated with better accuracy than CTP and MELD scores [17]. Moreover, ALBI was used evaluate survival in patients with decompensated cirrhosis receiving Direct Acting Antiviral (DAA) therapy in a study published as an abstract [18]

PALBI model was developed by incorporating platelet count into ALBI grade and was proposed to have better value in evaluating patient's survival with HCC [19] and recently has been used as a predictor of survival in patients with complications of liver cirrhosis [20]

\section{Aim of the study:}

To evaluate the predictive value of different patients' characteristics and risk scores as regard patients' outcome (death or survival).

\section{Patients and Methods}

This retrospective single center study was conducted between 2015 and 2018 on all patients admitted to the ward of Hepatology and Gastroenterology Department of the National Liver Institute, Menofia University, Egypt with complications of end stage liver diseases.

The study started after its approval by the institution review board of the National Liver Institute and obtaining and informed consent from patients enrolled in this study.

\section{Patients' selection:}

Patients with decompensated liver cirrhosis due to chronic Hepatitis C (HCV) infection were included in this study. The diagnosis of HCV infection based on sero-positivity of its antibodies or detection of any level of HCV viremia by Polymerase Chain Reaction (PCR) test.

The diagnosis of liver cirrhosis depended on presence clinical features of liver cirrhosis (e.g. history of liver disease, imaging features of cirrhosis, endoscopic findings of portal hypertension or patients' laboratory data). Liver decompensation was considered when patients presented with ascites, variceal bleeding, hepatic encephalopathy or non-obstructive hepatocellular jaundice.

Patients were enrolled in this study at time of their admission to hospital with one or more of the following complications:

I- Upper Gastro-Esophageal Bleeding (UGIB): When they presented with hematemesis and/or melena with presence of esophageal with/out gastric varices on upper endoscopy.

II- Hepatic encephalopathy based on clinical judgment and classified according to West-Haven criteria.

III- Spontaneous Bacterial Peritonitis (SBP) based on counting of ascitic fluid leucocytes' count $\left(<250\right.$ neutrophils $/ \mathrm{mm}^{3}$ and culture result of ascitic samples.

IV- Hepatorenal Syndrome (HRS): In those with progressive increase of serum creatinine even after stopping of diuretics and plasma volume expansion by infusion of human albumin at dose $(1 \mathrm{gm} . / \mathrm{kg})$ for 2 days.

V-Ascites: With different degrees with absence of any of previously mentioned complications. 


\section{Exclusion criteria:}

We excluded patients with liver diseases due to other etiologies e.g. chronic HBV, auto-immune or cholestatic liver diseases and alcoholic patients. We also excluded patients with acute liver failure, septic shock, those with hepatocellular carcinoma or other extra-hepatic malignancies and those $<18$ years old.

\section{Baseline assessment of the patients:}

On admission, patients' demographics were recorded including age, gender, etiology and duration of liver disease, other co-morbidities specially diabetes mellitus, history of previous admissions and previous similar attacks of the same presenting complication.

Routine laboratory work-up was done including liver function tests (serum albumin, bilirubin, transaminases [aspartate $\&$ alanine], alkaline phosphatase, INR and total protein levels), kidney function tests (serum creatinine, blood urea and serum sodium and potassium levels) and complete blood count (hemoglobin, white blood cells and platelets count).

Imaging study was done by routine ultrasound examination of the abdomen and dynamic computed tomography was requested on detecting any suspicious liver lesion. Upper endoscopy was done according to standard protocol for patients with upper gastro-esophageal bleeding. Therapeutic variceal band ligation was done for those with bleeding esophageal varices and sclerotherapy with tissue adhesives was done for bleeding gastric varices. Patients with refractory bleeding were referred for either Tran-sjagular Intrahepatic Portosystemic Shunt (TIPS) or underwent devascularization surgery. Ascitic fluid sampling was done under complete sterile conditions for those with ascites. Counting of total leucocytes and neutrophils and culture of these samples was done.

Evaluation for liver transplantation started for all patients according to the standard protocol of pre-transplant assessment in our institute. But, as Living Donor Liver Transplant (LDLT) is the only available type of liver transplantation in Egypt, many patients died during their evaluation for LDLT mostly due to scarcity of suitable donor.

\section{Study design:}

During admission, patients were treated according to the standard protocol for each complication which could be modified according to patients' response. The final outcome was stratified as survived or died at the end of their hospital admissions and this was considered as the dependent variable to be evaluated by this study.

In order to predict patients' mortality during hospitalization, different scores were calculated according to their known equations by using patients' data. MELD score was calculated according to the standard formula as $3.78 \mathrm{X} \mathrm{LN}$ (bilirubin $[\mathrm{mg} / \mathrm{dL}])+9.57 \mathrm{X} \mathrm{LN}($ creatinine $[\mathrm{mg} / \mathrm{dL}])+11.20$ X LN (international normalized ratio) + 6.43 [10] Minimal value for MELD score was 1 and maximal value for creatinine was 4 . Creatinine was set at 4 if the patient was receiving renal replacement therapy.

The MELD-Na score was calculated as MELD $+(140-\mathrm{Na}[\mathrm{mmol} / \mathrm{L}])-0.025$ X MELD X $(140$ - Na [mmol/L]) [14]. The Na concentration is bound between 125 and $140 \mathrm{mmol} / \mathrm{L}$. The conventional CTP score was calculated on the basis of serum bilirubin and albumin levels, the prothrombin time, as well as the presence and severity of ascites and encephalopathy [8]

The ALBI grade was calculated as follows: $[(\log 10$ bilirubin X 0.66) + (albumin X [-0.085]), where bilirubin is in 1 and albumin in $\mathrm{g} / \mathrm{L}]$ [16].

Accordingly, PALBI grade was calculated as the following equation: $2.02 \mathrm{X} \log 10$ bilirubin $0.37 \mathrm{X}(\log 10 \text { bilirubin })^{2}-0.04 \mathrm{X}$ albumin -3.48 $\mathrm{X} \log 10$ platelets $+1.01 \mathrm{X}(\log 10 \text { platelets })^{2}$ bilirubin was expressed in mollamin in $\mathrm{g} / \mathrm{L}$ and blood platelet count in 1000/

\section{Statistical analysis:}

Continuous variables were presented as mean \pm standard deviation and Categorical variables were expressed as frequencies or percentages. Comparisons of variables were performed using Student's $t$ for continuous variables and chi-square test for categorical variables. Multivariable Cox's proportional hazard model was carried out to identify independent factors associated with the outcome (death or survival). Factors with $p<0.05$ in the univariate cox regression analysis were entered into the multivariable model.

The discriminative ability of all studied prognostic scores to predict the outcome of patients with decompensated cirrhosis was evaluated by using the area under the receiver operating characteristic curve (ROC). This has the true-positive and false-positive rates on the vertical and horizontal axes, respectively. When the AUC approaches 1.0 , the model approaches $100 \%$ sensitivity and 
specificity. $p$-value $<0.05$ was considered statistically significant. Statistical analysis was conducted by SPSS (version 23.0 IBM).

\section{Results}

This study was conducted on 672 patients admitted to hospital with complications of end stage decompensated liver cirrhosis attributed to chronic $\mathrm{HCV}$ infection. Among these patients, 182 patients died during their hospital stay with overall hospital mortality rate of $(27 \%)$.

Table (1), shows demographics of studied patients at time of their admissions and their relation to patient's survival or death on univariate analysis.
Three hundred and fourteen patients presented with upper gastrointestinal bleeding of them 69 patients $(22 \%)$ died during their hospitalization. Also, 295 patients presented with hepatic encephalopathy either alone or with other complications (47.8\% mortality), 246 patients had SBP (40.2\% mortality) and 489 patients presented had ascites (34.7\% mortality) during hospitalization.

Univariate analysis of different laboratory parameters and their relation with patient's survival revealed that many variables (patients' age, serum total and direct bilirubin, albumin, transaminases, alkaline phosphatase, INR, serum creatinine, BUN, serum $\mathrm{Na}$ and white cells count) were significant with mortality $(p<0.05)$, Tables $(1,2)$.

Table (1): Patients' demographics on admission to hospital and its significance with hospital mortality.

\begin{tabular}{|c|c|c|c|c|}
\hline Variables $(\mathrm{N})$ & $\begin{array}{l}\text { Total } \\
\text { N. }(\%)\end{array}$ & $\begin{array}{l}\text { Survived } \\
\text { N. }(\%)\end{array}$ & $\begin{array}{c}\text { Died } \\
\text { N. }(\%)\end{array}$ & $\begin{array}{c}p- \\
\text { value }\end{array}$ \\
\hline \multicolumn{5}{|l|}{ Gender: } \\
\hline Male & $517(76.9)$ & $369(71.4)$ & $148(28.6)$ & \\
\hline Female & $155(23.1)$ & $121(78.1)$ & $34 \quad(21.9)$ & 0.100 \\
\hline UGIB on admission & $314(46.7)$ & $245(78)$ & $69(22)$ & $0.000 * *$ \\
\hline \multicolumn{5}{|l|}{ Frequency of UGIB attacks: } \\
\hline First attack & $142(45)$ & $105(73.9)$ & $37(26.1)$ & $0.007 * *$ \\
\hline Recurrent attack & $172(55)$ & $140(81.4)$ & $32(18.6)$ & \\
\hline \multicolumn{5}{|l|}{ Size of esophageal varices: } \\
\hline Small & $84 \quad(27)$ & $68 \quad(80.9)$ & $16(19.1)$ & $0.016^{*}$ \\
\hline Large & $230(73)$ & $177(76.9)$ & $53(23.1)$ & \\
\hline Presence of gastric varices & $120(38)$ & $108(90)$ & $12(10)$ & $0.000 * *$ \\
\hline \multicolumn{5}{|c|}{ Hepatic encephalopathy on admission: } \\
\hline Yes & $295(43.9)$ & $154(52.2)$ & $141(47.8)$ & $0.000 * *$ \\
\hline No & $377(56.1)$ & $336(68.5)$ & $41 \quad(22.5)$ & \\
\hline \multicolumn{5}{|l|}{ Grade of $H E$ : } \\
\hline Mild grades & $71 \quad(34.6)$ & $50(70.4)$ & $21(29.6)$ & $0.000 * *$ \\
\hline Severe grades & $134(65.4)$ & $69(51.5)$ & $65(48.5)$ & \\
\hline \multicolumn{5}{|c|}{ Diagnosis of SBP on admission: } \\
\hline Yes & $246(36.6)$ & $147(59.8)$ & $99(40.2)$ & 0.120 \\
\hline No & $426(63.4)$ & $343(80.5)$ & $83(19.5)$ & \\
\hline \multicolumn{5}{|c|}{ Presence of ascites on admission: } \\
\hline Yes & $498(74.1)$ & $325(65.3)$ & $173(34.7)$ & $0.012 *$ \\
\hline No & $174(25.9)$ & $165(94.8)$ & $9 \quad(5.2)$ & \\
\hline Hepatorenal syndrome & $171(25.4)$ & $87(50.8)$ & $84(49.1)$ & $0.023 *$ \\
\hline \multicolumn{5}{|l|}{ Ascites degree: } \\
\hline Mild ascites & $85 \quad(12.6)$ & $67 \quad(78.8)$ & $18 \quad(21.2)$ & $0.000 * *$ \\
\hline Moderate ascites & $320(47.6)$ & $198(61.9)$ & $122(38.1)$ & \\
\hline Marked ascites & $93 \quad(13.8)$ & $60 \quad(64.5)$ & $33 \quad(35.5)$ & \\
\hline \multicolumn{5}{|l|}{ CTP score grade: } \\
\hline A & $52 \quad(7.7)$ & $51 \quad(98.1)$ & (1.9) & $0.000 * *$ \\
\hline B & $116(17.3)$ & $110(94.8)$ & $6 \quad(5.2)$ & \\
\hline $\mathrm{C}$ & $504(75.0)$ & $329(65.3)$ & $175(34.7)$ & \\
\hline Total & $672(100)$ & $490(72.9)$ & $182(27.1)$ & \\
\hline
\end{tabular}


Table (2): Clinical and laboratory characteristics of studied patients and comparison between different risk scores.

\begin{tabular}{|c|c|c|c|c|}
\hline Variable & $\begin{array}{c}\text { Total }(\mathrm{N}=672) \\
\text { Mean } \pm \mathrm{SD} \text { (range) }\end{array}$ & $\begin{array}{c}\text { Survived } \\
(\mathrm{N}=490) \\
\text { Mean } \pm \mathrm{SD}\end{array}$ & $\begin{array}{c}\text { Died } \\
(\mathrm{N}=182) \\
\text { Mean } \pm \text { SD }\end{array}$ & $\begin{array}{c}p- \\
\text { value }\end{array}$ \\
\hline Age & $52.2 \pm 10.3(17-82)$ & $51.6 \pm 10$ & $53.7 \pm 10.9$ & $0.022 *$ \\
\hline MELD score & $19.2 \pm 7.9(6-48)$ & $16.7 \pm 5.6$ & $26.9 \pm 8.5$ & $0.000 * *$ \\
\hline CTP score & $11.2 \pm 2.7(5-15)$ & $10.5 \pm 2.5$ & $13.2 \pm 1.8$ & $0.000 * *$ \\
\hline MELD-Na score & $23.7 \pm 8.4(6-50)$ & $20.9 \pm 6.8$ & $31.7 \pm 7.4$ & $0.000 * *$ \\
\hline ALBI score & $-1.4 \pm 0.8(-3.4-0.6)$ & $-1.5 \pm 0.6$ & $-0.9 \pm 0.8$ & $0.000 * *$ \\
\hline PALBI score & $-2.2 \pm 0.8(-4.8-0.2)$ & $-2.4 \pm 0.7$ & $-1.7 \pm 0.8$ & $0.000 * *$ \\
\hline Duration of hospital stay & $7.3 \pm 5.3(1-48)$ & $7.2 \pm 5$ & $7.5 \pm 5.8$ & 0.451 \\
\hline Total bilirubin & $5.6 \pm 7.9(0.2-57)$ & $3.7 \pm 5.3$ & $10.7 \pm 109$ & $0.000 * *$ \\
\hline Direct bilirubin & $3.3 \pm 5.6(0.1-38)$ & $1.9 \pm 3.8$ & $6.9 \pm 7.7$ & $0.000 * *$ \\
\hline Total protein & $6.2 \pm 1.1(3-10)$ & $6.2 \pm 1.1$ & $6.1 \pm 1.1$ & 0.063 \\
\hline Albumin & $2.3 \pm 0.6(1-7.6)$ & $2.4 \pm 0.6$ & $2.0 \pm 0.5$ & $0.000 * *$ \\
\hline AST & $95.8 \pm 366.4(2-6697)$ & $63.5 \pm 70.2$ & $182.9 \pm 688.3$ & $0.021 *$ \\
\hline ALT & $66.4 \pm 144.3(2-2945)$ & $49.4 \pm 43.6$ & $112.1 \pm 262.9$ & $0.002 * *$ \\
\hline Alkaline phosphatase & $109.9 \pm 78.4(2-957)$ & $100 \pm 55.4$ & $136.6 \pm 116.2$ & $0.000 * *$ \\
\hline GGT & $53.3 \pm 65.5(1-978)$ & $52.6 \pm 67.8$ & $55.1 \pm 58.9$ & 0.637 \\
\hline INR & $1.9 \pm 0.79(1-9.53)$ & $1.7 \pm 0.5$ & $2.3 \pm 1.2$ & $0.000 * *$ \\
\hline BUN & $75.0 \pm 57.7(4-397)$ & $57.9 \pm 35.1$ & $121.3 \pm 77.9$ & $0.000 * *$ \\
\hline Creatinine & $1.4 \pm 1.1(0-15.4)$ & $1.1 \pm 0.5$ & $2.1 \pm 1.7$ & $0.000 * *$ \\
\hline $\mathrm{Na}$ & $130.2 \pm 7.7(93-145)$ & $132.2 \pm 6.3$ & $125 \pm 8.8$ & $0.000 * *$ \\
\hline $\mathrm{K}$ & $5.1 \pm 8.1(2-9.8)$ & $4.8 \pm 3.5$ & $5.9 \pm 14.4$ & 0.311 \\
\hline Hemoglobin & $10.0 \pm 2.2(3-19)$ & $9.9 \pm 2.1$ & $10.3 \pm 2.5$ & 0.057 \\
\hline WBCs & $10.1 \pm 7.2(1.4-69)$ & $8.8 \pm 5.9$ & $13.5 \pm 8.9$ & $0.000 * *$ \\
\hline Platelet count & $113.3 \pm 80.9(6-652)$ & $112.6 \pm 84.3$ & $115.1 \pm 71.6$ & 0.704 \\
\hline
\end{tabular}

Multivariate analysis for these significant variables revealed that age, total bilirubin, serum creatinine, blood urea nitrogen (BUN), serum sodium (Na), INR and white blood cells count were statistically significant with hospital mortality, $(p<0.05)$.

On comparing different scores as regard their discriminative abilities of predicting patient's mortality, MELD, MELD-Na and CTP score had better Area Under Receiver Operating Curve (AUROC) than AlBI and PALBI grades (AUROC >0.8). MELD-Na had slightly better AUROC than MELD score $(0.85$ vs. 0.84$)$.

PALBI and ALBI scores had similar AUROC but PALBI score had better accuracy even more than MELD score in predicting patient's mortality, Table (3) and Fig. (2).

The distribution of studied patients at different MELD score's categories is shown in Fig. (1). Patients were stratified into 6 categories according to their MELD scores: $<10,10-14,15-19,20-24$, 25-35 and >35. The mean value of MELD score was $19.2 \pm 7.9$ for all studied patients, while mean MELD was different between patients who survived than those who died (16.7 \pm 5.6 and $26.9 \pm 8.5$ respectively). The hospital mortality increased significantly across different MELD score categories with 70 patients $(84.3 \%)$ with MELD score $>35$ died during hospitalization, $(p<0.001)$, Table (2) and Fig. (1).

Table (3): Comparison between ROC characteristics as regard prediction of hospital mortality.

\begin{tabular}{lccccccccc}
\hline Score & AUC & $\begin{array}{c}\text { Cut off } \\
\text { level }\end{array}$ & $\begin{array}{c}\text { Sensitivity } \\
\%\end{array}$ & $\begin{array}{c}\text { Specificity } \\
\%\end{array}$ & $\begin{array}{c}\text { Odds } \\
\text { ratio\% }\end{array}$ & $\begin{array}{c}\text { Risk } \\
\text { ratio\% }\end{array}$ & $\begin{array}{c}\text { PPV } \\
\%\end{array}$ & $\begin{array}{c}\text { NPV } \\
\%\end{array}$ & $\begin{array}{c}\text { Accuracy } \\
\%\end{array}$ \\
\hline MELD score & 0.84 & 16 & 83 & 69 & 19.06 & 4.91 & 78 & 84 & 83 \\
CTP score & 0.82 & 11 & 80 & 67 & 11.65 & 4.26 & 69 & 84 & 80.8 \\
MELD-Na & 0.85 & 26 & 97 & 73 & 9.8 & 5.1 & 77 & 86 & 80.0 \\
ALBI grade & 0.75 & -1.5 & 91 & 78 & 7.73 & 1.9 & 68 & 67 & 79 \\
PALBI grade & 0.75 & -2.5 & 91 & 84 & 7.87 & 1.99 & 57 & 89 & 88 \\
\hline
\end{tabular}




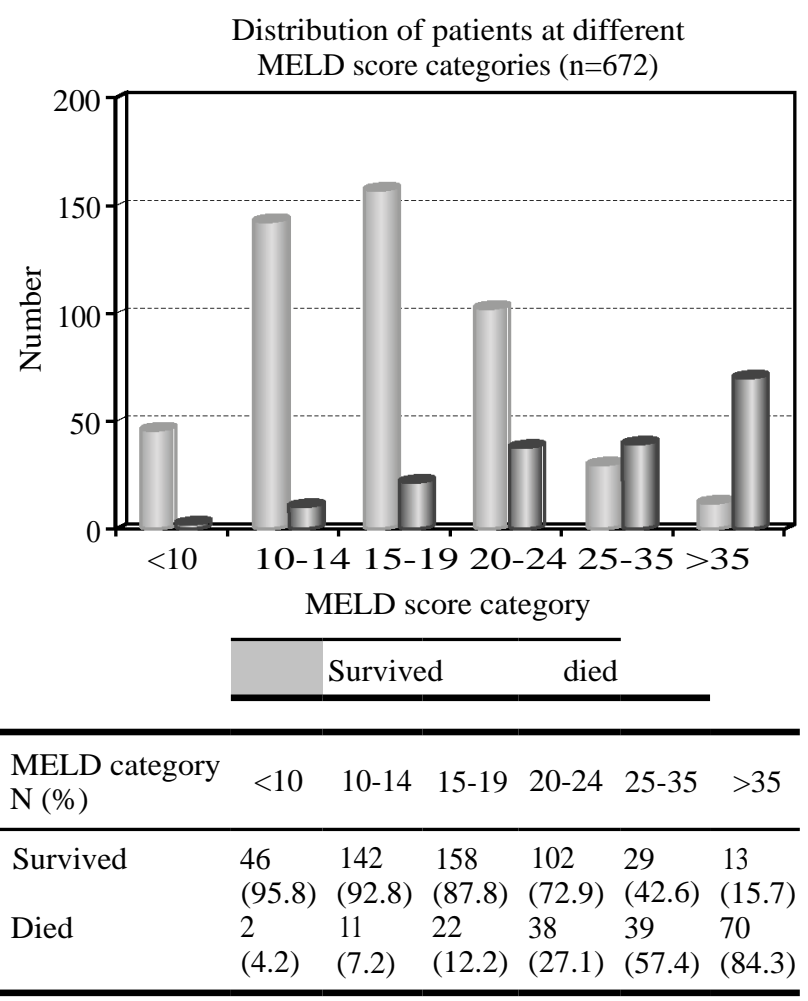

Fig. (1): Distribution of studied patients at different MELD score categories.
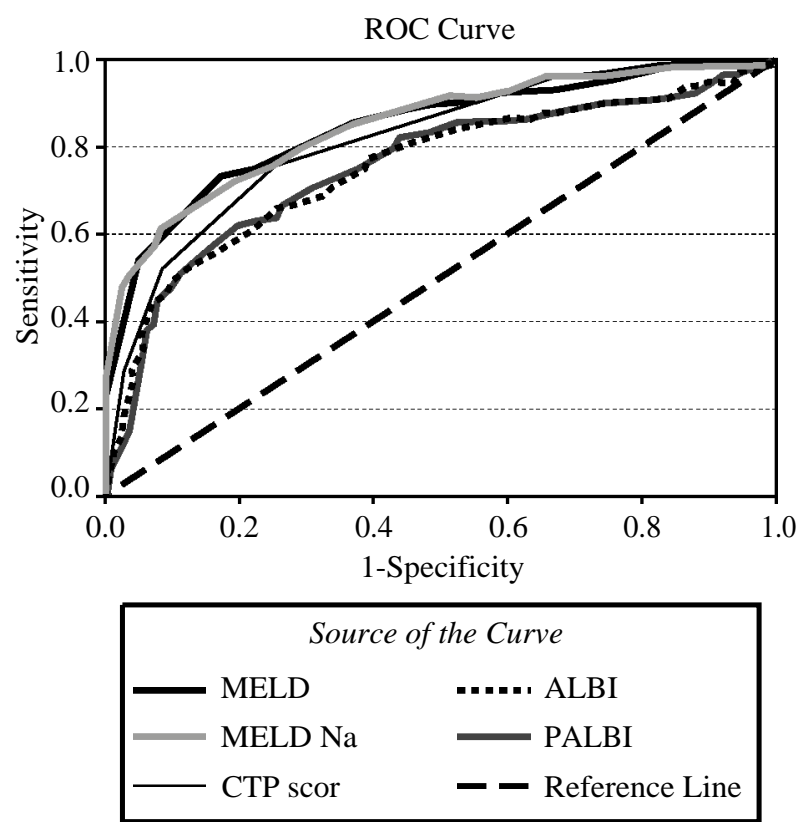

Fig. (2): ROCs of different scores as regard hospital mortality.

\section{Discussion}

In this study, we evaluated 672 patients with decompensated liver cirrhosis due to $\mathrm{HCV}$ infection presented with different complications (gastrointestinal bleeding, hepatic encephalopathy, SBP, HRS and ascites) in order to evaluate the predictive capabilities of different patients' characteristics and risk scores as regard patients' outcome (death or survival).

Over time, there was many attempts to develop a classification system which can characterize the degree of liver injury and predict prognosis of patients with liver cirrhosis using objective clinical and laboratory variables [7]

MELD and CTP scores are the main scores used for the prediction of prognosis of patients with liver cirrhosis. Peng et al, reviewed the diagnostic accuracy of MELD and CTP scores in a large meta-analysis and concluded that there's a need to develop a new score that can precisely predict prognosis of patients with liver cirrhosis [21].

Accordingly, MELD score was modified by adding serum sodium to its variables [14,15]. More recently, Johnson, et al., created a novel score, the ALBI grade, using only two biochemical parameters, serum albumin and total bilirubin levels. This was initially developed to assess liver condition in patients with HCC.

Studies had validated this new score in assessment of prognosis and defining management of patients with HCC [23-26]. Subsequently, studies validated ALBI grade for patients with chronic liver diseases in a study conducted on patients with upper gastrointestinal bleeding [27].

PALBI grade was developed in 2015 by Sasan, et al. [19] by incorporation of platelet count into ALBI grade to predict prognosis of patients with HCC undergoing curative interventions. Later on, it was validated on patients presented with complications of liver cirrhosis [28].

In this study, we aimed to evaluate predictors of prognosis of patients presented with complications of decompensated liver cirrhosis secondary to $\mathrm{HCV}$ infection.

The presence of such complications itself and frequency of its attacks were significant with hospital mortality. This agreed with many studies which confirmed that presence of such complications (e.g. UGIB, HE, SBP, HRS and ascites) is associated with increased mortality of such patients $[2,29,30]$

On correlating individual patients clinical and laboratory variables with mortality, patient's age, serum total and direct bilirubin, albumin, transaminases, alkaline phosphatase, INR, serum creatinine, BUN, serum Na and white cells count were significant with mortality, $(p<0.05)$. This agrees with 
many studies assuming that deterioration of liver and kidney functions and hyponatremia are strongly associated with mortality in patients with end stage liver disease [31-33]

To evaluate the predictive power of different scores on predicting prognosis of patients with complicated liver cirrhosis, univariate analysis showed strong statistical significance of MELD, MELD-Na, CTP scores, ALBI and PALBI grades with hospital mortality of these patients $(p<0.001)$.

So, for further comparison of discriminative power of these scores, ROCs of these scores were plotted and their AUROC, sensitivity, specificity and accuracy were calculated.

The AUROC versus the hospital mortality for these scores was not much different between the MELD (AUC: 0.84) vs. MELD-Na (AUC: 0.85), or CTP score (AUC: 0.82). But, this was higher than ALBI (AUC: 0.75) and PALBI grades (AUC: $0.75)$. But, PALBI grade had better accuracy in predicting mortality (88\%).

This was different from another study when ALBI grade was evaluated in 626 patients with decompensated cirrhosis due to HCV during DAA therapy and it was assumed that ALBI grade may be an independent risk factor for clinical deterioration and death [18]

In a study by Oikonomou et al., ALBI grade was evaluated prospectively in a large cohort of patients with stable decompensated cirrhosis of different etiology. They assumed that ALBI grade had discriminative ability (AUC: 0.695), better than CTP score (AUC: 0.611) and rather comparable to that of MELD score (AUC: 0.72), regarding the long-term outcome (death or LT) [34].

They also examined PALBI on a cohort of 325 stable decompensated patients which revealed that PALBI was an important risk factor (HR: 2.06, $95 \%$ CI [1.47-2.9], $p<0.001)$ associated with patients' outcome [34].

When PALBI grade was validated by Liu et al., it had good prognostic power in differentiating patients' survival (higher AUC values compared with MELD, CTP, and ALBI grade, all $p<0.05$ ) [28].

In another study, the PALBI had better discriminative ability for short term mortality than CTP score (AUC: 0.847 vs. 0.672, $p<0.001$ ) [20]

Our explanation of such result is that these large number of these patients with end stage liver disease had increased serum creatinine which increased the predictive power of MELD score and in addition to low serum sodium which also affected MELD-Na predictive power. These patients had ascites, hepatic encephalopathy and coagulopathy which markedly increased CTP score predictive capability for short term mortality.

Patients with MELD score $>35$ had the worst survival rate among patients ( 70 of them died $(84.3 \%)$ during hospitalization, $p<0.001)$. This was similar to a study by Ling et al., who found that patients with MELD > 39 before liver transplantation carried the worst survival [35]

Finally, we confirm there are restrictions of this study as it is a single center study and patients due to other etiologies should be included. Also, ALBI and PALBI grades are valuable scores which can predict prognosis of patients with decompensated cirrhosis but there's need of further researches to be confirmed.

\section{Conclusion:}

MELD-Na and MELD scores are good predictors for prognosis of patients with decompensated liver cirrhosis. But, ALBI and PALBI grade are promising scores which needs more validation.

\section{References}

1- JOHNSON K.B., CAMPBELL E.J., CHI H., ZHENG H., KING L.Y., WU Y., et al.: Advanced disease, diuretic use, and marital status predict hospital admissions in an ambulatory cirrhosis cohort. Dig. Dis. Sci., 59: 174-82, 2014.

2- SERAJ S.M., CAMPBELL E.J., ARGYROPOULOS S.K., WEGERMANN K., CHUNG R.T. and RICHTER J.M.: Hospital readmissions in decompensated cirrhotics: Factors pointing toward a prevention strategy. World J. Gastroenterol., 23: 6868-76, 2017.

3- CHIRAPONGSATHORN S., TALWALKAR J.A. and KAMATH P.S.: Readmission in cirrhosis: A growing problem. Curr. Treat. Options. Gastroenterol., 14: 23646, 2016.

4- BLACHIER M., LELEU H., PECK-RADOSAVLJEVIC M., VALLA D.C. and ROUDOT-THORAVAL F.: The burden of liver disease in Europe: A review of available epidemiological data. J. Hepatol., 58: 593-608, 2013.

5- FAYAD L., NARCISO-SCHIAVON J.L., LAZZAROTTO C., RONSONI M.F., WILDNER L.M., BAZZO M.L., et al.: The performance of prognostic models as predictors of mortality in patients with acute decompensation of cirrhosis. Ann. Hepatol., 14: 83-92, 2015.

6- PLANAS R., MONTOLIU S., BALLESTE B., RIVERA M., MIQUEL M., MASNOU H., et al.: Natural history of patients hospitalized for management of cirrhotic ascites. Clin. Gastroenterol. Hepatol., 4: 1385-94, 2006.

7- SCHUPPAN D. and AFDHAL N.H.: Seminar Liver cirrhosis. Lancet, 371: 838-51, 2008. 
8- PUGH R., MURRAY-LYON I. and DAWSON J.: Transection of the oesophagus for bleeding oesophageal varices. Br. J. Surg., 60: 646-9, 1973.

9- DURAND F. and VALLA D.: Assessment of the prognosis of cirrhosis: Child-Pugh versus MELD. J. Hepatol., 42: 100-7, 2005.

10- KAMATH P.S., WIESNER R.H., MALINCHOC M., KREMERS W., THERNEAU T.M., KOSBERG C.L., D'AMICO G., et al.: A model to predict survival in patients with end-stage liver disease. Hepatology, 33: 464-70, 2001.

11- BIGGINS S.W., RODRIGUEZ H.J., BACCHETTI P., BASS N.M., ROBERTS J.P. and TERRAULT N.A.: Serum sodium predicts mortality in patients listed for liver transplantation. Hepatology, 41: 32-9, 2005.

12- HEUMAN D.M., et al.: Persistent ascites and low serum sodium identify patients with cirrhosis and low MELD scores who are at high risk for early death. Hepatology, 40: 802-10, 2004.

13- MYERS R.P., et al.: Validation of the five-variable Model for End-stage Liver Disease (5vMELD) for prediction of mortality on the liver transplant waiting list. Liver Int., 34: 1176-83, 2014.

14- KIM W.R., BIGGINS S.W., KREMERS W.K., WIESNER R.H., KAMATH P.S., BENSON J.T., EDWARDS E., et al.: Hyponatremia and mortality among patients on the liver-transplant waiting list. N. Engl. J. Med., 359: 101826, 2008.

15- VITALE A., BERTACCO A., GAMBATO M., D'AMICO F., MORALES R.R., FRIGO A.C., ZANUS G., et al.: Model for end-stage liver disease sodium and survival benefit in liver transplantation. Transpl. Int., 26: 138-44, 2013.

16- JOHNSON P.J., BERHANE S., KAGEBAYASHI C., SATOMURA S., TENG M., REEVES H.L. and O'BEIRNE J., et al.: Assessment of liver function in patients with hepatocellular carcinoma: A new evidence based approach-The ALBI grade. J. Clin. Oncol., 33: 5508,2015 .

17- CHEN R.C., CAI Y.J., WU J.M., WANG X.D., SONG M., WANG Y.Q., ZHENG M.H., et al.: Usefulness of albumin-bilirubin grade for evaluation of long-term prognosis for hepatitis B-related cirrhosis. J. Viral. Hepat., 24: 238-45, 2017.

18- WALKER A.J., JOHNSON P.J. and BERHANE S.: Liver function as measured by albumin-bilirubin score within decompensated patients receiving direct acting antiviral therapy. In: The International Liver Congress 2017. Amsterdam, the Netherlands 19-23 April; 2017.

19- ROAYAIE P.S., JIBARA G., BERHANE S., TABRIZIAN P., PARK J.W., YANG J., YAN L., et al.: PALBI-An Objective Score Based on Platelets, Albumin \& Bilirubin Stratifies HCC Patients Undergoing Resection \& Ablation Better than Child's Classification. In AASLD Liver Learning; 2015.

20- ELSHAARAWY O., SAMEA E.A., GOMAA A., ALLAM N. and SAAD M.: PALBI, The Platelet-Albumin-Bilirubin score-A better predictor of outcome of Acute Variceal
Bleeding. In: The International Liver Congress 2017. Amsterdam, the Netherlands 19-23 April, 2017.

21- PENG Y., QI X. and GUO X.: Child-Pugh versus MELD Score for the Assessment of Prognosis in Liver Cirrhosis. Medicine (Baltimore), 95: 1-29, 2016.

22- CHAN A.W.H., KUMADA T., TOYODA H., TADA T., CHONG C.C.N., MO F.K.F., YEO W., et al.: Integration of albumin-bilirubin (ALBI) score into Barcelona Clinic Liver Cancer (BCLC) system for hepatocellular carcinoma. J. Gastroenterol. Hepatol., 31: 1300-6, 2016.

23- CHAN A.W.H., CHONG C.C.N., MO F.K.F., WONG J., YEO W., JOHNSON P.J., YU S., et al.: Incorporating Albumin-Bilirubin grade into the Cancer of the Liver Italian Program system for hepatocellular carcinoma. J. Gastroenterol. Hepatol., 32: 221-8, 2016.

24- KUO Y.H., WANG J.H., HUNG C.H., RAU K.M., WU I.P., CHEN C.H., KNEE K.M., et al.: The ALBI grade predicts the prognosis of patients with advanced hepatocellular carcinoma received sorafenib. J. Gastroenterol. Hepatol., 11, 2017.

25- OGASAWARA S., CHIBA T., OOKA Y., SUZUKI E., KANOGAWA N., SAITO T., MOTOYAMA T., et al.: Liver function assessment according to the AlbuminBilirubin (ALBI) grade in sorafenib-treated patients with advanced hepatocellular carcinoma. Invest. New Drugs, 33: 1257-62, 2015.

26- ZOU D., QI X., ZHU C., NING Z., HOU F., ZHAO J., PENG Y., et al.: Albumin-bilirubin score for predicting the in-hospital mortality of acute upper gastrointestinal bleeding in liver cirrhosis: A retrospective study. Turk. J. Gastroenterol., 27: 180-6, 2016.

27- LIU P.H., HSU C.Y., HSIA Y., LEE Y.H., CHIOU Y.Y., HUANG Y.H., LEE F.Y., et al.: ALBI and PALBI grade predict survival for HCC across treatment modalities and BCLC stages in the MELD Era. J. Gastroenterol. Hepatol., 32: 879-86, 2017.

28- SCAGLIONE S.J., METCALFE L., KLIETHERMES S., VASILYEV I., TSANG R., CAINES A., et al.: Early hospital readmissions and mortality in patients with decompensated cirrhosis enrolled in a large national health insurance administrative database. J. Clin. Gastroenterol., 51: 839-44, 2017.

29- VOLK M.L., TOCCO R.S., BAZICK J., RAKOSKI M.O. and LOK A.S.: Hospital readmissions among patients with decompensated cirrhosis. Am. J. Gastroenterol., 107: 247-52, 2012.

30- BIGGINS S.W., KIM W.R., TERRAULT N.A., et al.: Evidence-based incorporation of serum sodium concentration into MELD. Gastroenterology, 130: 1652e1660, 2006.

31- RUF A.E., KREMERS W.K., CHAVEZ L.L., et al.: Predicts Waiting List Mortality Better than. Vol. 11. 2005: $336 \mathrm{e} 343$.

32- ELIZABETH L. GODFREY a, MICHAEL L. KUEHT b, ABBAS RANA and SAMIR AWAD: MELD-Na (the new MELD) and peri-operative outcomes in emergency surgery. The American Journal of Surgery, 216: 407-13, 2018. 
33- OIKONOMOU, IOANNIS GOULIS, PETROS DOUMTSIS, THEODORA TZOUMARI, et al.: ALBI and PALBI Grades Are Associated with the Outcome of Patients with Stable Decompensated Cirrhosis. Annals of hepatology, 18 (1): 126-13, 2019.
34- LING Q., HAOJIANG D., RUNZHOU Z., TIAN S., WEILIN W., et al.: Predicting short-term survival after liver transplantation on eight score systems: A national report from China Liver Transplant Registry. Nature, 7: 42253 | DOI: 10. 103 8/srep42253, 2017.

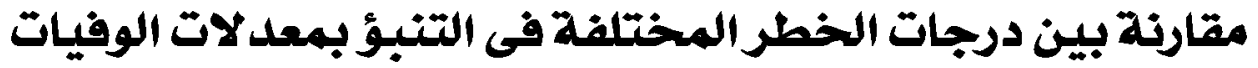

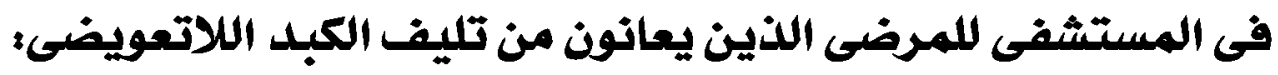

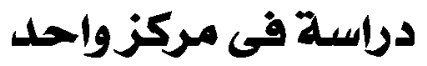

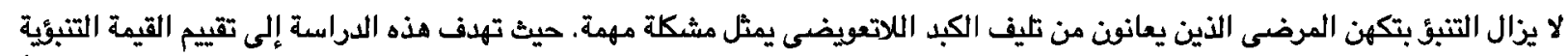

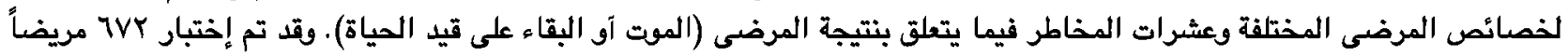

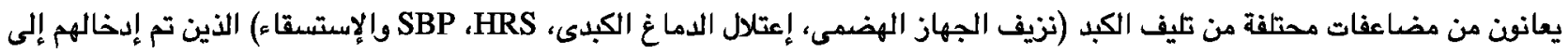

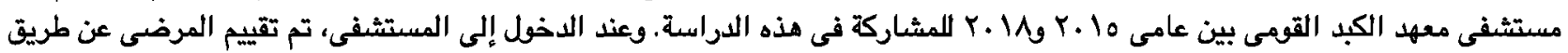

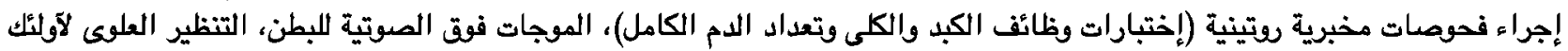

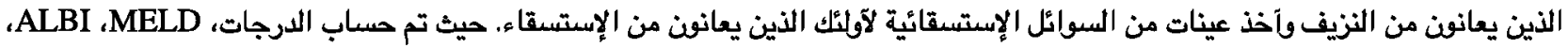

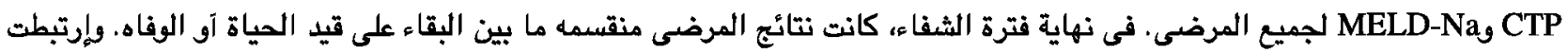

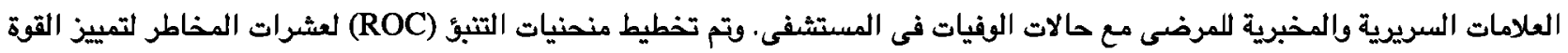

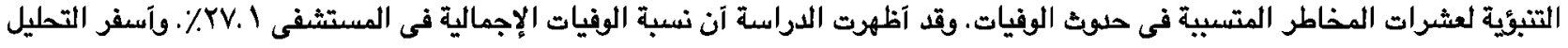

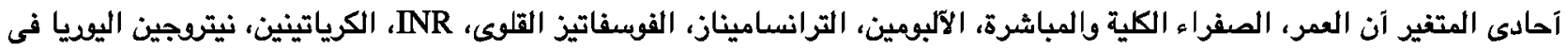

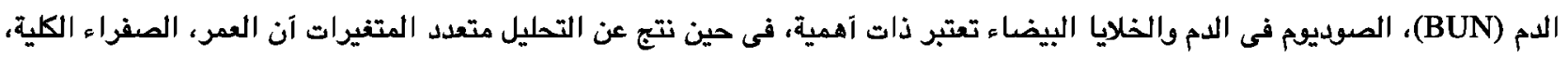

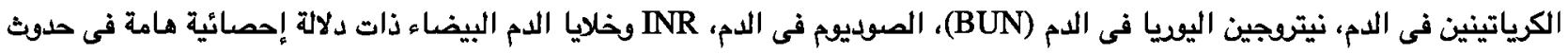

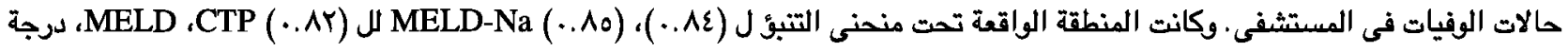
grade (.vo) PALBIg ALBI (. vo)

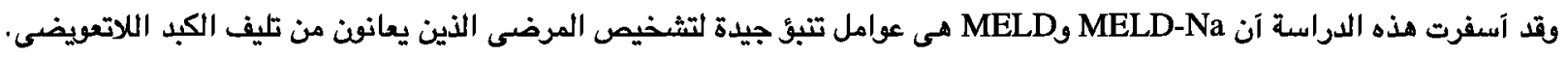
ولكن درجة PALBI ALBI هى علامات تتبؤ مستحدثة حيث تحتاج إلى مزيد من التحقق من دقتها. 